\title{
LO QUE PIENSAN DE LA ENSEÑANZA Y LO QUE HACEN LOS PROFESORES UNIVERSITARIOS
}

Carlos Manterola*

RESUMEN: Este trabajo pretende identificar las "teorías explícitas" e "implícitas" que poseen profesores universitarios sobre la enseñanza y apreciar si existen diferencias entre ellas. Para examinar las teorías implícitas se observaron y grabaron dieciséis horas de clases de 6 profesores, que se transcribieron y analizaron. Para identificar las teorías explícitas se les pasó un cuestionario, cuyas preguntas reflejan las teorías de la enseñanza. Un estudio estadístico simple por la frecuencia de las respuestas del cuestionario reflejó las teorías que dicen mantener los profesores. El análisis de las grabaciones del discurso del profesor en sus clases se efectuó en dos fases; una separando el discurso por actividad didáctica para conformar la unidad de análisis; y, la segunda, caracterizando cada actividad con códigos que permitieran ubicarlas en alguna de las cinco teorías de la enseñanza seleccionadas. Se concluye que los profesores "dicen" mantener con preferencia las teorías activa y constructiva, mientras que en su práctica de clase reflejan preferentemente una teoría tradicional con rasgos de la técnica y activa. La contradicción entre lo que se defiende verbalmente y lo que se hace no podía ser más neta. A la luz de estos resultados, se debe repensar la concepción de la formación de los docentes.

Palabras clave: Teorías Implícitas; Educación Superior; Enseñanza, Formación Docente.

\section{WHAT THEY THINK OF EDUCATION AND WHAT DO THE TEACHERS COLLEGE}

ABSTRACT: This paper aims to identify the "explicit theories" and "implicit" that have academics on teaching and assessing whether there are differences between them. To examine the implicit theories were observed and recorded sixteen hours of classes 6 teachers, which were transcribed and analyzed. To identify the explicit theories answered a questionnaire, whose questions reflected the theories of teaching. A simple statistical study by the frequency of questionnaire responses reflected the theories that say keep teachers. The analysis of the recordings of the speech of the teacher in the classroom was conducted in two phases: a speech by separating the educational activity to form the unit of analysis, and, second, characterizing each activity codes that can be positioned any of the five selected theories of teaching. It concluded that teachers "say" in preference to keep active and constructive theories, while in his classroom practice reflect preferably a traditional theory with features of the technique and active theories. The contradiction between what is defended orally and what is done could not be more distinct. In light of these results, we must rethink the concept of teacher training.

Keywords: Implicit Theories; Higher Education; Teaching; Teacher Training.

\footnotetext{
*Doutor em Educación. Profesor de Didácticas Especiales y Coordinador General de la Dirección de Extensión de la Escuela de Educación da Universidad Central de Venezuela (UCV) - Caracas E-mail:

manterolacarlos@yahoo.es
} 


\section{INTRODUCCIÓN}

Con la gran expansión de la Educación Superior a nivel mundial en la segunda mitad del siglo pasado, también se multiplicaron los desafíos a enfrentar, y así se manifiestan en la Declaración Mundial sobre la Educación Superior en el Siglo XXI: Visión y Acción (UNESCO, 1998) y se complementan en el $5^{\circ}$ Congreso Mundial de la Internacional de la Educación (IE), reunido en Berlín, en julio del 2007 cuando:

\footnotetext{
"Reconoce que los estudiantes de hoy (como trabajadores de mañana) necesitan niveles de Educación más altos y aptitudes más complejas para poder desenvolverse en un mercado laboral mundial tan competitivo ..." y, lo complementa con "la convicción del profesional docente, en el suministro de una Educación de alta calidad a todos los niveles,..." (p. 1)
}

La primera función de la Educación Superior radica en la necesidad de otorgar una formación integral cualificada a estudiantes que deben enfrentarse a un mundo cada vez más exigente, tanto en su capacitación profesional como en la formación de ciudadanos responsables para estar a la altura de los tiempos modernos. Y esta función es imposible llevarla a cabo sin profesionales de la docencia de alta calidad en todos los niveles, no solamente en el área de su especialidad sino además en su formación pedagógica y didáctica que lo hagan eficaz en ayudar a los estudiantes en sus aprendizajes.

La lectura de estos documentos internacionales le debe llevar a los centros de Educación Superior a colocar como primera línea de sus políticas de desarrollo y mejoramiento la formación didáctica de su profesorado.

\section{EL DESAFÍO AL CUAL NOS ENFRENTAMOS}

La psicología cognitiva presenta dos tipos de saberes: uno, el abstracto y formal -que suele ser producto de la escolarización- y que sirve para comprender las explicaciones sobre los fenómenos; el otro, el conocimiento que se origina en la Educación informal, en las experiencias personales del sujeto y que tiene una gran capacidad para solucionar las dificultades concretas con las que la persona se enfrenta en el día a día. Ambos conocimientos conforman la cultura de la persona. Al primer tipo de conocimientos, Rodrigo; Rodríguez; Marrero (1993), Pozo (1996), Pozo y otros (2006), lo denominan teorías explícitas y los segundos saberes conforman las llamadas teorías implícitas. Los conocimientos explícitos desarrollan en las personas la competencia del saber decir, mientras que los saberes implícitos son de naturaleza no consciente, dependientes del contexto específico y le aportan al sujeto eficacia en su hacer, más que en el decir (POZO et alli, 2006).

A esta diferenciación se agrega las afirmaciones que provienen de la neurociencia, y que privilegian la importancia del conocimiento informal sobre 
el explícito. En este sentido, el neurólogo Marcus Raichle dice: "La mayor parte de lo que vemos lo construye el cerebro" (Raichle, 2009, p 2), ya que parece -según sus palabras- que solamente el 10\% de las sinapsis que realiza el cerebro proceden del exterior, mientras que el resto procede del interior de la persona.

Si estos dos planteamientos los aplicamos al programa de formación pedagógica para los profesores, se puede aceptar - por un lado- que los docentes poseen una teoría explícita y consciente de la enseñanza y -simultáneamente- otra teoría, implícita e inconsciente sobre ella; y, -por el otro lado- que las teorías implícitas son mucho más importantes que las explícitas por cuanto inciden directamente sobre el quehacer docente.

¿Cómo se caracterizan estas teorías sobre la enseñanza que poseen los profesores? ¿El profesor enseña como dice que enseña o su acción es diferente a como la expresa? ¿Es diferente saber explicar cómo se debe enseñar a cómo se desarrolla la acción didáctica?

Estas preguntas llevaron a definir los objetivos de la investigación sobre las concepciones didácticas de los profesores; qué tipo de enseñanza defienden, cómo enseñan en la práctica laboral y, qué diferencias existen entre unas y otras.

\section{OBJETIVOS DE LA INVESTIGACIÓN}

El trabajo se propone únicamente tres objetivos que son los siguientes:

(1) Reconocer las teorías explícitas sobre enseñanza que manejan los profesores de Educación Superior

(2) Examinar las teorías implícitas sobre enseñanza de los profesores de Educación Superior

(3) Analizar las semejanzas y diferencias entre unas y otras.

\section{MARCO TEÓRICO QUE FUNDAMENTA LA INVESTIGACIÓN}

Este trabajo se ha desarrollado con el financiamiento del CDCH-UCV, que - a su vez- se inserta dentro de la línea investigativa denominada "Cambio Escolar y Mejoramiento de la Calidad en la Escuela" que se desarrolla en el Doctorado en Educación del Postgrado de la Facultad de Humanidades y Educación, de la Universidad Central de Venezuela.

En este trabajo, como se especificó en los objetivos, se van a analizar las teorías de enseñanza que maneja el docente, tanto en lo que dice como en lo que hace, para así poder repensar los programas de formación pedagógica del profesorado de Educación Superior.

Sí se han llevado a cabo, y no en número pequeño, trabajos sobre el pensamiento y las creencias de los docentes; por ejemplo, se han realizado inves- 
tigaciones sobre el pensamiento epistemológico de los educadores (Martín, Porlan, Rivero, 1998), sobre sus creencias educativas (Marcelo, 1987; Pajares, 1992; Córdova, 2006), sobre sus representaciones sobre el aprendizaje (Pozo, 1989, 1994, 2006; Sánchez, 2000; Freites, 2003) sobre las teorías implícitas que mantienen sobre diferentes conceptos educativos especialmente en la área de las ciencias naturales, sobre el Conocimiento Práctico (Elbaz, 1988, 1991; Connelly y Clandinin, 1985, 1990), sobre el Conocimiento Didáctico del Contenido (Shulman, 1987; Marcelo, 1997; Grossman, 2005) todo lo cual está constituyendo un amplio y fundamentado cuerpo de conocimientos sobre el conocimiento de los profesores (Putnam y Borko, 1998) (citado por Marcelo, 2005).

También se ha trabajado sobre las concepciones didácticas que utilizan los educadores durante su práctica laboral en los niveles preuniversitarios (Marcelo, 1995; Fernández y Elortegui, 1996; De Pro Bueno y otros, 2000; Latorre, 2004; Vega, 2005; Cochran, Zeichner y Fries, 2006; Medina y otros, 2006; Pozuelos, Travé y Cañal, 2007; Cañal, 2009; Manterola, 2010) y, en menor cantidad, en los profesores de Educación Superior (Gómez, 2003; de Ketele, 2003; Margalef, 2005; Alvarez, 2006; Bobadilla, Cárdenas, Dobbs y Soto 2009; Serrano, 2010). En estos trabajos se presentan varias clasificaciones sobre las teorías pedagógicas que los docentes poseen y utilizan en su labor didáctica, así como sobre los tipos de profesores en orden a su enseñanza.

En este trabajo, únicamente se van a mencionar cuatro autores que organizan las teorías pedagógicas, las cuáles concuerdan en el significado que se les da a las mismas, y que servirán de referencia teórica para analizar las creencias y concepciones didácticas de los profesores, que se realiza en este trabajo.

Por un lado Marrero (1993), después de un estudio histórico sobre las concepciones pedagógicas en la Educación durante los últimos cuatro siglos, distingue "cinco grandes corrientes pedagógicas: tradicional, activa, crítica, técnica y constructiva” (Marrero, 1993, p 246). Según este autor, la teoría tradicional de la enseñanza retoma la Educación centrada en la autoridad del profesor; la perspectiva activa, que pudiera estar representada por Rousseau y Dewey; la crítica, que se consolidará en el siglo XX con Giroux, Freire, Apple, Kemmis; la perspectiva técnica, fundamentada en los conductistas primero y con la teoría de sistemas después; y, por último, la teoría constructivista que, apoyándose también en Rousseau, se consolida con la obra de Piaget y los autores de la pedagogía operatoria.

A su vez, Pérez (1999), basándose en los “intereses rectores del conocimiento" señalados por Habermas (1982), utiliza cuatro perspectivas de análisis para comprender la acción profesional; éstas son: la racionalista, la técnica, la humanista y el pensamiento práctico.

Fernández y Elortegui (1996) caracterizan cinco modelos didácticos en los docentes que los denominan profesor transmisor cuya prioridad es el cumplimiento del programa; el profesor tecnológico, que organiza detalladamente la clase con objetivos claros dirigidos a adquirir los conocimientos y capacidades de la 
disciplina; el denominado profesor artesano, que suele ser más frecuente en los primeros niveles del sistema escolar; el profesor descubridor que basa su enseñanza en la investigación empírica por descubrimiento; y, por último, el modelo del profesor constructor, que se suele encontrar muy escasamente y vinculado a grupos de trabajo alrededor de un proyecto, en donde la perspectiva constructivista del aprendizaje está muy presente.

Pozo y otros (2006), estudia la enseñanza y el aprendizaje sobre la clasificación en tres posturas fundamentales: la teoría directa, la interpretativa y la constructiva, pero al final agregan una cuarta postura que no se atreven a denominarla teoría y la llaman la visión posmoderna (Torrado y Pozo, 2006). La teoría directa (TD) es la teoría implícita del aprendizaje y de la enseñanza más básica. La teoría interpretativa (TI) proviene de la evolución de la teoría directa; "es la que predomina en los modos en que aprendices y profesores dan cuenta del aprendizaje" (Pozo y otros, 2006, p 123). La teoría constructiva (TC) que "implica procesos mentales reconstructivos de las propias representaciones acerca del mundo físico, sociocultural e incluso mental, así como de autorregulación de la propia actividad de aprender". Y, por último, la llamada visión posmoderna que asume una postura relativista radical, según la cual no habría ninguna posibilidad de evaluar las distintas representaciones del conocimiento.

\section{METODOLOGÍA EMPLEADA EN LA INVESTIGACIÓN}

En la descripción de este punto, se va seguir las tres dimensiones de la metodología de la investigación que plantea Dendaluce (1999): la epistemológica, la metodológica y la técnica.

La dimensión epistemológica de esta investigación se determina en base al modelo "interpretativo y constructivista", utilizando la nomenclatura de Pérez (1992, 1997, 1999), que "se preocupa fundamentalmente por indagar el significado de los fenómenos educativos en la complejidad de la realidad natural donde se producen" (Pérez, 1999:28). En concreto, se analizan los discursos de los profesores durante sus clases, los cuáles son grabados en audio.

La dimensión propiamente metodológica contempla el tipo y alcance de la investigación. Este trabajo, bajo el criterio del origen de la fuente de datos, se corresponde con un estudio de campo y no con una investigación documental; de acuerdo al criterio de las intenciones del investigador, este trabajo se puede catalogar fundamentalmente como descriptivo, aunque se inicia el análisis al categorizar el discurso de los profesores.

La dimensión técnica considera los tipos de datos y los procesos de análisis de los mismos. En esta investigación se utilizan dos procedimientos para la recolección de los datos y dos formas de análisis de ellos. Para el primer objetivo, que consiste en reconocer las teorías explícitas sobre la enseñanza que los profesores dicen mantener, se utiliza el cuestionario de Marrero (1993). Un cues- 
tionario conformado por 30 preguntas tipo likert para cubrir los aspectos más importantes de las cinco teorías didácticas: tradicional, activa, técnica, constructiva y crítica, que el autor plantea, distribuyendo 6 ítems por teoría. Se trabajó con seis profesores universitarios que dan clases de Química a los cuales se les pasó el cuestionario mencionado. Para identificar las teorías implícitas de los profesores sobre la enseñanza -segundo objetivo de la investigación- se les observó sus clases y se grabó el discurso de los profesores mientras enseñaban. En total se grabaron 16 horas académicas de clase, distribuidas en 8 sesiones; en concreto al Profesor 1 y 2 se les grabó a cada uno 4 horas de clase, y a los Profesores 3, 4, 5 y 6 se les grabó a cada uno dos horas de clase. El cuestionario y la observación y grabación de clases se realizó entre los meses de mayo y septiembre del 2009.

De los seis profesores, dos tienen el título de Doctor, uno es Magister y otro cursa la Maestría y los dos más jóvenes son licenciados, todos ellos en la mención de Química.

La selección de los docentes no es aleatoria, sino que se fue conformando en la medida en que se tenía la oportunidad de contactar con ellos y no pusieran indisposición para ser grabados y responder el cuestionario.

Adicionalmente se pasó el cuestionario a 40 profesores más de Educación Superior. El análisis de los datos del cuestionario se realizó con un estudio descriptivo estadístico por la frecuencia de respuesta.

El análisis del discurso de los profesores mientras daban sus clases se realizó en dos niveles. En el primero se divide el discurso del docente por tipo de actividad didáctica que realiza; por ejemplo: si el profesor presenta los objetivos de la clase, si explica el contenido, si amenaza, si manda tarea, etc. Es decir, en este primer nivel de análisis se describe la secuencia de actividades que realiza el profesor durante la clase; es decir, nos da la estructura didáctica de la clase.

En el segundo nivel del análisis, se estudia cada actividad y se le atribuye códigos (significado didáctico de la actividad) que responden a las características que conforman cada una de las teorías sobre la enseñanza. Por medio de estos códigos, se puede llegar a inferir la teoría didáctica que predomina en la clase del profesor y la presencia en mayor o menor grado de otras teorías didácticas (ver en anexo, la tabla n. 1).

\section{RESULTADOS DEL ANÁLISIS DE LOS DATOS}

\section{Resultados del Cuestionario}

Las respuestas del cuestionario se analizaron tomando en cuenta tres aspectos (Pérez y Gimeno, 1990): el primero seleccionando las proposiciones con mayor aceptación por el colectivo de los profesores (40 docentes); el segundo estudiando las proposiciones con mayor rechazo por parte del profesorado y, 
por último, el tercero, analizando por teorías de la enseñanza las posturas que dicen mantener los profesores. Este último análisis se hará también con los seis profesores a los que se les observó las clases para después poder hacer la comparación entre su "decir" y su "hacer".

(A) En primer lugar, siguiendo a Pérez y Gimeno (1990) se seleccionaron las diez proposiciones del cuestionario que poseen mayor aceptación por parte de los profesores y aquellas con mayor rechazo (ver en anexo Tabla 2). Al analizarlas, se aprecia que hay tres proposiciones (V15, V4 y V12) que defienden una comunicación abierta en la clase, en la que se mantiene el derecho a la discusión y la libertad de participar en clase, lo cual -a su vez- coincide con el rechazo a la proposición V9, que obtiene la menor puntuación.

Así mismo se aprecia una concordancia de pareceres en las respuestas a las preguntas V14, V19 y V3 en donde se defiende la importancia de fijarse en el desarrollo permanente del alumno y no únicamente en los resultados finales.

En las respuestas a las preguntas V13 y V24 se considera la interrelación que existe y debe existir entre escuela y sociedad.

Mientras que en las dos que tienen menor puntuación de las más preferidas (V2 y V24), nos indican una tendencia al centralismo en el profesor de conducir el ritmo de la clase, y la manifestación técnica de tomar en cuenta las necesidades para tener éxito.

(B) Una vez analizadas las proposiciones por su significado directo, se evaluan las proposiciones de acuerdo a las teorías de la enseñanza que representa cada una de ellas. Para ello, se hace una sumatoria de los valores obtenidos por las proposiciones seleccionadas con el mayor acuerdo, clasificadas por la teoría de la enseñanza que representan. El resultado nos dice (ver anexo tabla n. 3) que las cinco teorías están presentes en la opinión de los profesores, existiendo muy poca diferencia en los valores promedios de cada una de las cinco teorías. El orden de preferencia fue el siguiente: teoría activa $(23,14 \%)$, constructiva $(21,86 \%)$, crítica $(18,74 \%)$, tradicional $(18,81 \%)$ y, por último, la teoría técnica $(17,55 \%)$. La teoría de la enseñanza más representativa para los 40 docentes es la teoría activa que está representada por un 23,04\%, mientras que la teoría técnica que constituye la menos representada alcanza a un $17,55 \%$; es decir que hay muy poca diferenciación entre las teorías. Lo que pudiera indicar que existen criterios de diferenciación muy débiles entre lo que dicen los profesores sobre la enseñanza.

Al hacer este mismo análisis con los seis profesores de nuestro estudio se consiguen resultados muy semejantes (ver anexo Tabla n. 4). En todos ellos, están presentes las cinco teorías de la enseñanza, con pequeñas diferencias de porcentaje entre ellas.

Como se aprecia, son muy semejantes las respuestas dadas a los cuestionarios por los seis profesores analizados en sus clases y por el colectivo de los 40 profesores de Educación Superior. Están presentes las seis teorías y casi en porcentaje de representación muy similar. La teoría activa sigue siendo la teoría 
con mayor representatividad en los seis profesores, aunque el profesor 6 mantiene una teoría constructivista de la enseñanza con un poco mayor de representatividad que la teoría activa. En los seis profesores la teoría crítica es la que tiene menor presencia y las teorías activa y constructivista las que poseen mayor puntaje.

\section{Resultados del Análisis del Discurso del profesor}

Como se explicó en el apartado de la metodología, el análisis del discurso del profesor se realizó en dos niveles. Uno apreciando la secuencia de actividades distintas que desarrollaba durante la clase y el otro que consistió en darle significado didáctico al tipo de actividad realizada e identificada en el primer análisis (ver tabla n. 1).

Estos análisis se realizaron con las 8 clases de los seis profesores estudiados y dieron como resultado lo siguiente:

Las dos clases de Química analizadas del profesor 1 corresponden al primer semestre de la carrera con unos 40 estudiantes por sección; entre las dos clases observadas existe una semana de separación entre ellas. Su enseñanza está centrada en un modelo predominante durante toda la clase, en donde el profesor es el centro de la actividad de aula, en la que predomina la palabra como instrumento de comunicación, en la que utiliza como recurso didáctico unas veces el video beam con presentación power point y siempre la pizarra.

Los estudiantes todos van al mismo ritmo de estudio y no existe discusión sobre la bondad o consistencia del conocimiento que se trabaja. La enseñanza está basada en la disciplina y nunca existe una relación con la vida diaria o social de los alumnos.

El alumno siempre está ocupado y hay que aprovechar el tiempo. El aprendizaje se concibe que se alcanza por la repetición sucesiva y abundante de algoritmos. La única participación de algún alumno que se atreve a pasar a la pizarra consiste en seguir las orientaciones que le da el docente para resolver el ejercicio.

Aunque hay un ambiente comunicativo distendido, cada cierto tiempo el profesor actúa para evidenciar que la autoridad del salón radica en él.

Pudiéramos decir que estas características pertenecen a una concepción de la enseñanza denominada tradicional, que es la que predomina en la clase del profesor 1 .

Sin embargo también aparecen algunos pocos rasgos de la teoría técnica y activa, tales como la preocupación por la búsqueda de los resultados correctos de los ejercicios, por lo que se va a preguntar en los exámenes y por las referencias no escasas a éstos. También se aprecia la postura técnica de la enseñanza en la preocupación por no perder el tiempo, por presentar ordenadamente el trabajo y por la búsqueda de la claridad en el contenido. La actividad permanente en que se mantiene toda la clase pudiera ser una característica pro- 
pia de la teoría activa. Sin embargo no se aprecian en el profesor 1 rasgos de las teorías constructivista ni de la crítica en su quehacer didáctico.

El profesor 2 da clases en el tercer semestre y las dos clases observadas con una diferencia de una semana entre ellas son muy similares a las del profesor 1. Difiere en algo del profesor 1 por su tendencia a intensificar el aspecto de la exposición magistral, que posteriormente la alterna con las preguntas puntuales a los estudiantes como recursos para seguir su explicación. Lleva las clases más estructuradas y ordenadas y se apoya siempre en presentaciones power point que las presenta por video beam y conducen el hilo didáctico. Por supuesto el centralismo del profesor es muy grande. La enseñanza se sigue concentrando en los contenidos disciplinares, los cuales pareciera que no tiene relación con otras cosa sino con la misma química. Este profesor 2 se maneja entre estas dos corrientes la técnica y la tradicional, aunque siempre tiene rasgos de la activa. No se aprecian tampoco características de las teorías de la enseñanza constructiva ni de la crítica.

Los profesores 3, 4, 5 y 6 fueron observados solamente en una clase de una duración de dos horas académicas cada una.

El Profesor 3 sigue la pauta didáctica de los profesores 1 y 2. Es una clase magistral, apoyado en presentaciones por video beam, que de vez en cuando utiliza la pregunta como recurso para seguir con su explicación verbal. Es una clase centrada en la disciplina, sin relaciones con la vida del alumno ni del entorno social en el que nos movemos. Se busca la claridad y la eficacia en la enseñanza siguiendo una planificación didáctica ordenada. Por tanto, el análisis de su discurso nos dice que posee rasgos dominantes de la teoría técnica combinada con características de la teoría tradicional. Siempre apoyado en algún aspecto de la teoría activa.

El Profesor 4 no se diferencia de los anteriores. Su didáctica está totalmente centrada en él, que se manifiesta en el protagonismo que sigue en la clase y la no participación de los alumnos. Este protagonismo le lleva a utilizar ese estilo de enseñanza muy utilizado de preguntar y responder; pero pregunta no para que le respondan sino para él conducir la dinámica de la clase.

De igual manera se muestra que se enseña la Química sin salirse de la Química; no hay referencias a la vida del alumno, de la comunidad, del mundo. Lo que busca el docente es lograr que los alumnos aprendan el algoritmo de balancear y hacer los cálculos estequiométricos correspondientes. No se trata de formar al estudiante; consiste, más bien, en aprender un algoritmo. También se observa que el docente utiliza el estilo de explicar, en este caso apoyándose en el libro, y después hacer ejercicios. Haciendo más y más ejercicios iguales se logra el aprendizaje del algoritmo. Se mantiene, pues, la misma posición sobre la enseñanza. Una enseñanza con grandes rasgos de la teoría Tradicional y de la enseñanza Técnica.

El Profesor 5 es más joven que los anteriores y tiene una relación de mayor camaradería con los alumnos. De igual manera utiliza analogías con el 
mundo social y cercano al estudiante que le pueden ayudar a entender. Sin embargo priva el orden en la planificación de la clase y la calidad de los objetivos didácticos. Domina también la clase magistral, la explicación centrada en el profesor ayudado por las presentaciones en video beam. No se sale de la disciplina que se muestra aislada de todo otro saber. El éxito por los resultados y la enseñanza de algoritmos están fuertemente representados en su práctica didáctica. Por todo ello se puede decir que domina una teoría sobre la enseñanza de tipo técnica combinada con la tradicional y en algo la activa.

El Profesor 6, por último, también sigue la clase magistral, centrada en él y en los contenidos de la disciplina. No pierde tiempo, busca la claridad. Pero sí utiliza con mayor frecuencia las analogías con la vida real de los alumnos, lo cual favorece la creación de un clima de cierta camaradería. El orden en la exposición, los objetivos que se buscan están claros y pretende tener toda la eficacia posible en lograr los aprendizajes. Pudiéramos resumir que también predomina la teoría técnica combinada con la tradicional.

En resumen, la estructura de las ocho clases (16 horas) analizadas de Educación Superior siguen un mismo patrón con pequeñas diferencias entre ellos. El patrón didáctico está constituido por las clases magistrales que se interrumpen por las preguntas que periódicamente realiza el profesor con el ánimo más de mantener el orden y la atención de los estudiantes que de entablar alguna discusión sobre el tema. También estas preguntas tienen el sentido de interrumpir momentáneamente el discurso y utilizarlo como descanso, para seguir inmediatamente otra vez con la explicación del profesor. Este patrón se mantiene ya se explique un tema como cuando se resuelven ejercicios. Es el docente el protagonista aunque sea el estudiante el que está en el pizarrón haciendo el ejercicio, pues cumple el papel de copiador en la pizarra de lo que dice el docente. Este comportamiento es el habitual y el dominante durante toda la clase, de ahí que sea la teoría de la enseñanza tradicional la que más se utiliza en el quehacer del profesor.

También es frecuente el rasgo de llevar bastante bien organizada la clase en base a la estructura de la asignatura, pues se aprecia que se domina el contenido disciplinar y se sigue un orden en la explicación; ese orden no responde a un planteamiento didáctico sino - como se dijo- a la estructura de la materia que se imparte. Este rasgo está muy unido al criterio de no perder el tiempo, de mantener ocupados a los alumnos. En tal sentido, se puede decir que también se manifiesta la teoría de la enseñanza técnica con algún atisbo de teoría activa.

En ninguno de los seis profesores aparece ningún rasgo que los ubique en las teorías constructivista, ni crítica. 


\section{Comparación de las teorías explícitas e implícitas de los profesores sobre la enseñanza}

Si revisamos la Tabla n. 4, se aprecia que los profesores analizados de Educación Superior dicen que están de acuerdo con rasgos de las cinco teorías de la enseñanza, que las mantienen con muy poca diferencia entre ellas.

Las teorías activa y constructiva son las más representadas en su opinión, recogida en los cuestionarios pero, y los valores más pequeños de aceptación atañe a las teorías tradicional y técnica. Sin embargo, como se decía más arriba, las diferencias entre las cinco teorías es muy pequeña. Hasta aquí las teorías explícitas sobre enseñanza manifestadas por los profesores.

Sin embargo, como se ha visto en el análisis del discurso del profesorado, grabado en la clase, se puede decir que existen dos teorías representadas en sus clases: las teorías tradicional y la técnica, siendo la primera la más sobresaliente con bastante diferencia sobre la segunda.

La diferencia, pues, es notable, entre las teorías explícitas e implícitas del profesorado de Educación Superior, entre lo que dicen mantener y lo que hacen en sus clases. Dicen mantener - por medio del cuestionario- una amalgama casi igualitaria de las cinco teorías con un pequeño predominio de la teoría activa y constructiva sobre las demás, mientras que en su quehacer didáctico utilizan fundamentalmente la teoría tradicional y técnica, estando ausente la teoría constructivista y apenas se manifiesta tímidamente en algunos profesores la teoría activa.

Por lo que se puede concluir que existe una gran disparidad entre lo que se piensa sobre la enseñanza y lo que se practica. Se piensa como un profesor defensor de la teoría activa y constructivista y se practica la labor docente como un profesor tradicional y técnico.

\section{A MODO DE CONCLUSIÓN}

Es urgente revisar la práctica didáctica del profesorado de la Educación Superior por cuanto está alejada de toda consideración teórica pedagógica moderna y de toda reflexión sobre la misma práctica. Es la misma disciplina que se imparte (la Química) y su epistemología las que determinan las actividades didácticas y su secuencia definiendo así el sentido que deben tener en la formación de los estudiantes. Esta es la denominada "didáctica disciplinar", que se constituye ya como categoría organizacional en el seno del conocimiento científico (Morin, 1990).

Esta didáctica que predomina en la Educación Superior está fundamentada en varios soportes que le han dado fortaleza y dominio en el tiempo, habiendo conformado una concepción pedagógica en la que la transmisión de contenidos disciplinares, no siempre vigentes, es el eje que dirige la enseñanza.

Los soportes que fundamentan esta didáctica disciplinar son la enseñanza planificada y ejecutada por un docente, en un aula, con un contenido dis- 
ciplinar, durante una o dos horas de clase con unos 37 alumnos que estudian y se evalúan individualmente. Si separamos las variables de su definición, nos queda más claramente expuestos los seis pilares sobre los que se apoya la "didáctica disciplinar":

Un docente que planifica, ejecuta y evalúa el trabajo escolar El aula como espacio privilegiado para el aprendizaje

La enseñanza centrada en la transmisión de los conceptos y teorías de la disciplina

El tiempo de trabajo se divide en fracciones de 45 ó 90 minutos

Se trabaja con secciones de 30 a 40 estudiantes

El trabajo es individual y la evaluación mide los conocimientos alcanzados

Este baluarte se manifiesta en clases centradas en el conocimiento indiscutible del profesor, que se distribuye de igual manera aunque el estudiantado sea muy diferente. Son clases magistrales, centradas en la asignatura, sin relación con la problemática social y personal de los alumnos, que se estudia generalmente no porque interesa sino porque es requisito indispensable para aprobar el examen. Una didáctica que transmite conceptos y teorías para que posteriormente sean devueltos de manera la más parecida posible por los estudiantes en el examen.

Ante esta situación, es fundamental que se revise la política de formación pedagógica y didáctica del profesorado de Educación Superior, que -además- no puede estar basada en talleres orales y explicativos sino en miniproyectos que exijan creaciones de acciones didácticas distintas. 


\section{ANEXOS}

\section{Tabla 1}

Ejemplo del análisis de las clases realizado en dos fases o niveles; primero separando el discurso por las actividades didácticas realizadas ( $2^{\mathrm{a}}$ columna) y posteriormente (última columna de la tabla) otorgando un significado didáctico a la actividad (código).

\begin{tabular}{|c|c|c|}
\hline $\begin{array}{l}\text { DISCURSO DEL } \\
\text { DOCENTE }\end{array}$ & $\begin{array}{l}\text { SECUENCIA DE } \\
\text { ACTIVIDADES }\end{array}$ & $\begin{array}{l}\text { SIGNIFICADO } \\
\text { DIDÁCTICO }\end{array}$ \\
\hline Prof.: Buenos Dias. & Saluda. & \\
\hline silencio por favor. & Poca ordem. & Autoridad del professor \\
\hline Hoy vamos a seguir conl guia de problemas. ¿Quién puede leer el problema numero 31 ? & $\begin{array}{l}\text { Informa el ejecicio que se } \\
\text { va hacer. }\end{array}$ & Centralismo del professor \\
\hline Alumna: La Escherichia colli es un bacilo intestinal que puede contener... & Lee alumna el ejercicio. & \\
\hline P.: Mas alto por favor. & Control del docente. & Autoridad del professor \\
\hline $\begin{array}{l}\text { Alumna: La Escherichia colli es un bacilo intestinal que puede contener } 1,66.10^{-14} \mathrm{~g} \text { de } \\
\text { ADN. Si lamassa molar del ADN és } 2,5.10^{9} \mathrm{~g} \text { de mol, la cantidad de moléculas de ADN } \\
\text { en cada uno de essos bacilos és de: } \\
\text { a) } 8 \\
\text { b) } 4 \\
\text { c) } 4.10^{18} \\
\text { d) } 3.10^{18} \\
\text { P.: Cuando los ellos hablan } 6,36.10^{14} \text { gramos,me hablan ¿de què? Me están piediendo } \\
\text { cantidad de moléculas de ADN, que será ese dato ¿Que creen ustedes }\left(6,36-10^{14}\right) \text { ? } \\
\text { Saben o no saben, }\end{array}$ & $\begin{array}{l}\text { Lee alumna el ejercicio } \\
\text { Inicia la explicación con } \\
\text { perguntas sobre la } \\
\text { compreensión del texto } \\
\text { leido. }\end{array}$ & Centralismo del professor \\
\hline $\begin{array}{l}\text { recuerden que el examen esta cerca. } \\
\text { Alumnos: no (respondem varios) } \\
\text { P.: No saben, okay. }\end{array}$ & \begin{tabular}{|l|} 
Amenaza com examem. \\
Ratifica el professor que \\
no saben.
\end{tabular} & Evaluar por exâmenes \\
\hline $\begin{array}{l}\text { Según los datos del problema estos } 6,36-10^{14} \text { gramos de ADN, me están dando una } \\
\text { masa, las unidades me lo están diciendo, ¿de qué es la masa? ¿Qué es el ADN? }\end{array}$ & Explica perguntando. & $\begin{array}{l}\text { Centralismo de professor. } \\
\text { Enfoque disciplinar }\end{array}$ \\
\hline
\end{tabular}

Alumnos: (nadie reponde)

Tabla 2

Las once proposiciones con el mayor acuerdo obtenido

\begin{tabular}{|c|c|c|}
\hline $\begin{array}{l}\text { NÚMERO DE LA } \\
\text { PERGUNTA }\end{array}$ & PROPOSICIONES & $\begin{array}{l}\text { ACUERDO } \\
\text { VALOR }\end{array}$ \\
\hline V15 & Estoy convencido/a de quelas relaciones en el aula deben ser plurales e iagulaes. & 4,55 \\
\hline V13 & $\begin{array}{l}\text { Creo que es necessario integrar la escuela al medio, solo así podemos preparar } \\
\text { a los alumnos para la vida. }\end{array}$ & 4,45 \\
\hline V14 & $\begin{array}{l}\text { Al evaluar opino que lo fundamental es valorar no solo el resultado, sino el conjunto de } \\
\text { actividades realizadas por el alumno. }\end{array}$ & 4,40 \\
\hline V4 & $\begin{array}{l}\text { Emmi opinión, la discussión en la classe es esencial para mantener una adecuada } \\
\text { actividad de enseãnza. }\end{array}$ & 4,40 \\
\hline V24 & $\begin{array}{l}\text { Soy plenamente consciente de que la enseñanza contribuye a la seleción, } \\
\text { preservación y transmissón de normas y valores explícitos u ocultos. }\end{array}$ & 4,40 \\
\hline V19 & $\begin{array}{l}\text { Suelo tenes en cuenta cuando evalúo si los trabajos elaborados por los alumnos van } \\
\text { evolucionando durante el curso. }\end{array}$ & 4,35 \\
\hline V3 & $\begin{array}{l}\begin{array}{l}\text { Suelo comprobar más el processo de aprendizaje de los alumnos que los } \\
\text { resultados finales. }\end{array}\end{array}$ & 4,28 \\
\hline V12 & $\begin{array}{l}\text { Procuro que, en mi clase, los alumnos estén continuamente opinando } \\
\text { y ocupados en algo. }\end{array}$ & 4,28 \\
\hline V23 & $\begin{array}{l}\text { Estoy convencido/a de que aquello que el alumno/a aprende por experimentación, } \\
\text { no lo olvida nunca. }\end{array}$ & 4,20 \\
\hline V2 & Procuro que todos mis alumnos sigan el ritmo que yo marco para la clase. & 4,18 \\
\hline V27 & $\begin{array}{l}\text { Siempre he dicho que, para que una escuela funcione de forma eficaz, hay que hacer } \\
\text { una adecuada valoración de necessidades. }\end{array}$ & 4,18 \\
\hline
\end{tabular}


Tabla 3

Teorías Explícitas del professorado de Educación Superior sobre la enseñanza

\begin{tabular}{c|c}
$\begin{array}{c}\text { TEORÍAS DE LA } \\
\text { ENSEÑANZA }\end{array}$ & $\begin{array}{c}\text { VALOR PROMEDIO } \\
\text { EN \% }\end{array}$ \\
\hline ACTIVA & 23,04 \\
\hline CONSTRUCTIVA & 21,86 \\
\hline CRÍTICA & 18,74 \\
\hline TRADICIONAL & 18,81 \\
\hline TÉCNICA & 17,55 \\
\hline TOTAL & $\mathbf{1 0 0}$
\end{tabular}

Tabla 4

Teorías Explícitas de los 6 professores analizados comparados con el otros 40 docentes en Educación Superior (datos en \%)

\begin{tabular}{|c|c|c|c|c|c|c|}
\hline & ACTIVA & CONSTRUCTIVA & CRÍTICA & TRADICIONAL & TÉCNICA & TOTAL \\
\hline PROFESSOR 1 & 25,12 & 21,62 & 17,62 & 18,68 & 18,65 & 100,00 \\
\hline PROFESSOR 2 & 23,03 & 19,69 & 17,20 & 18,98 & 18,06 & 100,00 \\
\hline PROFESSOR 3 & 26,26 & 19,19 & 17,17 & 19,19 & 18,18 & 100,00 \\
\hline PROFESSOR 4 & 24,78 & 22,12 & 17,7 & 16,81 & 18,58 & 100,00 \\
\hline PROFESSOR 5 & 24,37 & 22,69 & 17,65 & 17,65 & 17,65 & 100,00 \\
\hline PROFESSOR 6 & 20,72 & 21,62 & 17,12 & 21,62 & 18,92 & 100,00 \\
\hline $\begin{array}{c}\text { PROMEDIO } \\
\text { DE LOS } 6 \text { PROFESSORES }\end{array}$ & 24,03 & 21,41 & 17,41 & 18,82 & 18,33 & 100,00 \\
\hline $\begin{array}{c}\text { PROMEDIO } \\
\text { DE LOS } 40 \text { PROFESSORES }\end{array}$ & 23,04 & 21,86 & 18,74 & 18,81 & 17,55 & 100,00 \\
\hline
\end{tabular}




\section{BIBLIOGRAFÍA CITADA}

ALVAREZ, Fco. (2006) Saber pedagógico y formación docente. Disponible en: http://mt.educarchile.cl/MT/Falvarez/2006/09/saber_pedagogico_y_formacion_d.html- <Consulta 2007, diciembre $15>$

BOBADILlA G., M., CÁRDENAS P., A, DOBBS D.,E y SOTO B., A. M. (2009) “Los rodeos de la práctica". Representaciones sobre el saber docente en el discurso de estudiantes de Pedagogía. Estudios Pedagógicos XXXV(1): 239-252.

CAÑA, P. (2009) Activismo, enseñanza de las ciencias en Primaria y formación del profesorado. Investigación en la Escuela, 67:5-22.

COCHRAN-SMITH, M., ZEICHNER, K. y FRIES, K. (2006). Estudio sobre la formación del profesorado en los Estados Unidos: descripción del informe del comité de la American Educational Research Association (AERA) sobre investigación y formación del profesorado. Revista de Educación, 340:87-116.

COLL, C y SÁNCHEZ, E. (2008) Presentación. El análisis de la interacción alumno-profesor: líneas de investigación. Revista de Educación, 346:15-32

El $5^{\circ}$ Congreso Mundial de la Internacional de la Educación (IE), reunido en Berlín, Alemania, del 22 al 26 de julio de 2007. Disponible en: http://www.ei-ie.org/worldcongress2007/eiie/docs/Resolutions/2007-00195-01-S.pdf. Consulta 2008, febrero 15.

CÓRDOVA, D. (2006) El pensamiento pedagógico de los estudiantes de Educación. Revista de Pedagogía, 79: 231-269.

DE KETELE, Jean-Marie (2003). La formación didáctica y pedagógica de los profesores universitarios: luces y sombras. Revista de Educación, 331:143-169.

DE PRO, A., SAURA L., O y SÁNCHEZ B., G. (2000) ¿Qué actividades de enseñanza utilizan los profesores en formación inicial y los profesores en ejercicio cuando planifica unidades didácticas de ciencias? Investigación en la Escuela, 40:23-37.

DENDALUCE S., Iñaki (1999) La investigación educativa ante el tercer milenio. Bordón. v. 51, n. 4:363-376.

ELBAZ, F. (1988): “Cuestiones en el estudio del conocimiento de los profesores". En Villar Angulo, L. M.: Conocimientos, creencias y teorías de los profesores. Alcoy, Marfil, p. 88-96.

FERNÁNDEZ G., J y ELORTEGUI, N. (1996) Qué piensan los profesores de cómo se debe enseñar. Enseñanza de las Ciencias, 14(3):331-342.

FREITES, Migdalia. (2003) Enseñanza y Comunicación. Una alternativa para la formación docente. Trabajo especial para ascender a la categoría de Profesor Agregado. Universidad Central de Venezuela: Caracas.

GÓMEZ L. L. F. (2003) Las teorías implícitas de los profesores y sus acciones en el aula. Ponencia presentada en el VII Congreso Nacional de Investigación Educativa en Guadalajara, Jalisco (2111- 2003).

GROSSMAN, P., WILSON, S. SHULMAN, L. (2005) Profesores de sustancia: el conocimiento de la materia para la enseñanza. Profesorado. Revista de curriculum y formación del profesorado, 9(2).

HABERMAS, J. (1982). Conocimiento e Interés. Madrid:Taurus (original, 1972. Knowledge and Human Interests. Londres:Shapiro).

LATORRE N., M. (2004) Aportes para el análisis de las racionalidades presentes en las prácticas pedagógicas. Estud. Pedagógicos. 30:75-91. Disponible En:

$<$ http://www.scielo.cl/scielo.php?script=sci_arttext\&pid=s 0718 $07052004000100005 \& \operatorname{lng}=\mathrm{es} \& \mathrm{nrm}=\mathrm{iso}>$. Consulta 2005, marzo 15

MANTEROLA, C. (2003) Lo que se enseña y lo que se aprende: una perspectiva institucional. Tesis de Doctorado en Educación. Postgrado de la Facultad de Humanidades y Educación de la Universidad Central de Venezuela.

MANTEROLA, C. (2010) ¿Qué teorías determinan nuestra acción didáctica? Ponencia presentada en XII Jornadas de Investigación Educativa y III Congreso Internacional. Escuela de Educación, UCV, mayo 2010.

MARCELO, C. (1987) El pensamiento del profesor. Barcelona: CEAC. 
MARCELO, C. (1995) Formación del profesorado para el cambio educativo, Barcelona: PPU.

MARCELO, C. (1997) ¿Quién forma al formador?”. Revista de Educación, 313:249-278.

MARCELO, C. (2005) La investigación sobre el conocimiento de los profesores y el proceso de aprender a enseñar. Una revisión personal. En Perafán, G. A. y Adúriz-Bravo, A. (comp) Pensamiento y conocimiento de los profesores. Debate y perspectivas internacionales. Bogotá:Universidad Pedagógica Nacional/Nomos, (2 ed).

MARCELO, Carlos (2009) los comienzos en la docencia: un profesorado con buenos principios. Profesorado. Revista de curriculum y formación del profesorado, v. 13, n. 1.

MARGALEF G., L. (2005). La formación del Profesorado Universitario: análisis y evaluación de una experiencia. Revista de Educación, 337:389-402.

MARRERO, J. (1993) Las teorías implícitas del profesorado: vínculo entre la cultura y la práctica de la enseñanza, en Rodrigo, M.J., Rodríguez, A, y Marrero, J. (eds.) Las teorías implícitas. Una aproximación al conocimiento cotidiano. Madrid, Visor.

MARTÍN, Rosa, PORLÁN, Rafael, RIVERO, Ana (1998. Conocimiento profesionaly epistemología de los profesores, II: estudios empíricos y conclusiones. Disponible en:

http://dialnet.unirioja.es/servlet/oaiart?codigo $=94921<$ Consulta 2005, octubre 20>

MEDINA, M., TEJERA R., C., y FERNÁNDEZ G. J. (2006). Formación didáctica de profesores: experiencia de convergencia entre el profesorado de la Universidad y el de Educación secundaria. Investigación en la escuela, 58:93-102.

MORIN, E. (1990): Introducción al pensamiento complejo, Barcelona, Editora Gedisa.

PAJARES, F. (1992) Teachers' Beliefs and Educational Research: Cleaning Up a Messy Construct. Review of Educational Research, 62(3), 307-332.

PÉREZ G., A.I. (1992). Comprender la enseñanza en la escuela. Modelos metodológicos de investigación educativa. En Gimeno, J y Pérez, A.I. Comprender y Transformar la enseñanz̧a. Madrid:Morata, pp. 115-136.

PÉREZ G., A.I. (1997). Historia de una Reforma educativa. Sevilla:Díada

PÉREZ G., A.I. (1999). La cultura escolar en la sociedad neoliberal. Madrid:Morata, 2 ed. (1ª edición en 1998).

PÉREZ, A. y GIMENO, J. (1990). Pensamiento y acción del profesor: de los estudios sobre la planificación del pensamiento práctico. Revista de Pedagogía, 11, 22, 9-36.

POZO J.I.(1996). Aprendices y Maestros. Madrid: Alianza.

POZO, J. I. (1989). Teorías cognitivas del aprendizaje, Madrid: Morata.

POZO, J. I.(1994). Solución de problemas, Madrid: Santillana.

POZO, J. I., SCHEUER, N., MATEOS, M., PÉREZ E., Ma del Puy (2006) las teorías implícitas sobre el aprendizaje y la enseñanza. En Pozo, J. I y otros Nuevas Formas de Pensar la Enseñanza y el Aprendizaje. Madrid: Grao. pp. 95-132.

POZUELOS, F. F., TRAVÉ, G y CAÑAL, P. (2007) Acerca de cómo el profesorado de primaria concibe y experimenta los procesos de investigación escolar. Revista de Educación, 344:403-423.

RAICHLE, Marcus (2009) La vida privada del cerebro. REDES 32. Disponible en: http://www.redesparalaciencia.com/?s=Raichle\%2C+Marcus $+<$ Consulta 2009, mayo 30> RODRIGO M.J.; RODRÍGUEZ A.; MARRERO J., (1993) Las teorias implícitas. Madrid: Visor. SÁNCHEZ, L. (2000) Concepciones de aprendizaje del saber universitario y del saber popular. Tesis Doctoral. Facultad de Humanidades y Educación. Universidad Central de Venezuela.

SERRANO, Rocío C. (2010) Pensamientos del profesor: un acercamiento a las creencias y concepciones sobre el proceso de enseñanza-aprendizaje en la Educación Superior. Revista de Educación. n. 352.

SHULMAN, L. (1987) Knowledge and Teaching. Foundations of the New Reform. Harvard Educational Review, 57(1), traducido en Shulman, L. (2001) Conocimiento y Enseñanza. Estudios Públicos, 83

TORRADO, J. A y POZO, J.I (2006) Del dicho al hecho: de las concepciones sobre el aprendizaje a la práctica de la enseñanza de la música. En Pozo, J. I y otros. Nuevas Formas de Pensar la Enseñanza y el Aprendizaje. Madrid:Grao, pp. 205-228. 
Lo que piensan de la enseñanza y 10 que hacen los professores universitarios

UNESCO (1998). Declaración Mundial sobre la Educación Superior en el Siglo XXI: Visión y Acción. Disponible en: http://www.unesco.org/education/educprog/wche/declaration_spa.htm $<$ Consulta 2009, octubre 20>

VEGA G., L. (2005). Los sistemas educativos europeos y la formación de profesores. Los casos de Francia, Reino Unido, España y Finlandia. En: Revista de Educación, 336:169-187

Data recebimento: $14 / 06 / 2010$

Data aprovaçãa: 05/02/2011

Data versão final: $08 / 02 / 2011$ 
